\title{
Hydrodynamics in bridging and aggregation of two colloidal particles in a near-critical binary mixture
}

\author{
Shunsuke Yabunaka ${ }^{1}$, Ryuichi Okamoto $^{2}$, and Akira Onuki ${ }^{3}$ \\ ${ }^{1}$ Yukawa Institute for Theoretical Physics, Kyoto University, Kyoto 606-8502 Kyoto, Japan \\ ${ }^{2}$ Department of Chemistry, Tokyo Metropolitan University, Hachioji, Tokyo 192-0397, Japan \\ ${ }^{3}$ Department of Physics, Kyoto University, Kyoto 606-8502, Japan
}

(Dated: June 5, 2022)

\begin{abstract}
We investigate bridging and aggregation of two colloidal particles in a near-critical binary mixture when the fluid far from the particles is outside the coexistence (CX) curve and is rich in the component disfavored by the colloid surfaces. In such situations, the adsorption-induced interaction is enhanced, leading to bridging and aggregation of the particles. We realize bridging firstly by changing the temperature with a fixed interparticle separation and secondly by letting the two particles aggregate. The interparticle attractive force dramatically increases upon bridging. The dynamics is governed by hydrodynamic flow around the colloid surfaces. In aggregation, the adsorption layers move with the particles and squeezing occurs at narrow separation. These results suggest relevance of bridging in the reversible colloid aggregation observed so far. We use the local functional theory [J. Chem. Phys. 136, 114704 (2012)] to take into account the renormalization effect and the simulation method [Phys. Rev. Lett. 85, 1338 (2000)] to calculate the hydrodynamic flow around the colloidal particles.
\end{abstract}

\section{INTRODUCTION}

Colloidal suspensions exhibit various intriguing effects in a mixture solvent. For example, interplay between wetting and phase separation has been studied extensively in experiments $\underline{1}^{-\underline{\underline{4}}}$ and simulations $\mathrm{s}^{\underline{5}-\underline{7}}$. Much attention has also been paid to a solvent-mediated attractive interaction among solid surfaces due to adsorptioninduced concentration disturbances ${ }^{8-13}$, which is enhanced with increasing the correlation length $\xi$ near the criticality. In particular, reversible colloid aggregation has been observed at off-critical compositions outside the coexistence curve $(\mathrm{CX})^{14} \underline{22}$, where the solvent far from the particles is rich in the component disfavored by the colloid surfaces (near the disfavored branch of CX). It occurs while the particle radius $a$ still much exceeds $\xi$. Remarkably, the face-to-face separation distance $\ell$ is of order $a$ and is much longer than $\xi$ in these aggregates $14-19$. As a result, the aggregates fragment and the particles redisperse upon a reduction of adsorption caused by a small temperature change. Guo et al .21 furthermore observed liquid, fcc crystal, and glass phases of the aggregated particles also for $\ell \sim a \gg \xi$. We mention a number of theoretical papers on this near-critical aggregation $\underline{23}-30$. In non-critical solvents, on the other hand, the particles often stick and form fractal aggregates due to the attractive van der Waals interaction ${ }^{31}$.

The adsorption-induced interaction decays exponentially as $\exp (-\ell / \xi)$ for $\ell \gtrsim \xi$ if the solvent is at the critical composition without phase separation ${ }^{8.10,11}$, as was measured directly ${ }^{32}$. Some authors ${ }^{22.27}$ used this exponential expression to explain the observed aggregation. In their approach, close contacts of the particles in the range $\ell \lesssim \xi$ are needed for aggregation, since the factor $\exp (-\ell / \xi)$ is nearly zero for $\ell \gg \xi$. In contrast, the van der Waals two-body potential ${ }^{31,33}$ is long-ranged for $\ell>a$ and grows as $-A_{\mathrm{H}} a / 12 \ell$ for $\ell<a$, where $A_{\mathrm{H}}$ is the
Hamaker constant. In recent theories $\stackrel{28,29}{ }$, the colloidal particles were assumed to form one-component systems interacting via an attractive solvent-mediated interaction potential $\left(\propto e^{-r / \xi}\right)$ and a repulsive electrostatic potential, where the particle separation $r$ is smaller than $\xi$ in aggregation. Very recently, Edison et al $\underline{\underline{30}}$ has examined phase behavior of a three-component model on a 2D lattice to obtain aggregation of the third component.

As a related problem, much attention has also been paid to the phase behavior of fluids between closely separated walls ${ }^{34}-36$, where narrow regions may be filled with the phase favored by the walls or may hold some fraction of the disfavored phase. As a result, there can be a firstorder phase transition between these two states, called capillary condensation, when the fluid in the reservoir is in the disfavored phase. For fluids between parallel plates, there appears a first-order capillary condensation line outside (bulk) CX in the plane of the temperature $T$ and the reservoir chemical potential $\mu_{\infty}$ (which corresponds to a magnetic field for magnetic systems) $)^{8,34-38}$. Analogously, between large particles (or between a large particle and a wall), a bridging domain of the favored phase can appear, when they are surrounded by the disfavored phase $\mathrm{I1}^{11}, 39-\underline{45}$. This effect is relevant in the physics of wet granular matter $\underline{46}$. Recently, we calculated phase diagrams of capillary condensation ${ }^{47}$ and bridging 48 . We stress that the solvent-mediated interaction can be much enhanced near these transitions even in pretransitional states (before local phase separation) $)^{10,13,47,48,50}$. We also examined the dynamics of capillary condensation ${ }^{49}$.

In this paper, we demonstrate that the capillary force mediated by a bridging domain can give rise to the observed reversible aggregation. Let two colloidal particles be bridged by a columnar domain of the favored phase, where the column radius is of the order of the particle radius $a$. Then, in terms of the surface tension $\sigma$, the interface free energy is of order $2 \pi \sigma a \ell$ and its derivative 
with respect to $\ell$ yields the capillary force of order $2 \pi \sigma a$ between the particles $41,44,48$. Such bridging can occur even for large $\ell$ of order $a$, so it can be relevant to the reversible aggregation. We then need to investigate its dynamics to demonstrate its occurrence for $\ell \sim a \gg \xi$ near the disfavored branch of CX. It is a local phase separation process governed by the hydrodynamics, where squeezing also takes place as the particles approach. We note that the colloid hydrodynamics coupled with the concentration has been studied in various situations ${ }^{1,7,51}$ using the model $\mathrm{H}$ equations ${ }^{52}-54$.

As a theoretical method, we use the renormalized local functional theory $47,48,55-57$ to account for the nearcritical fluctuation effect. It is combined with the fluidparticle dynamics (FPD) simulation method 1,7,58,59 to calculate the flow around the particles. In our case, the adsorption layers are thickened near the criticality ${ }^{60}-62$, which strongly affect the flow in aggregation. We mention that Furukawa et al. ${ }^{51}$ used the FPD method to study two-particle aggregation at the critical composition. They assumed a small particle radius $a$ with $a \sim \xi$ in the mean-field theory, where the solvent-mediated force was appreciable even for $\ell \sim a$ because of relatively large $\xi$. However, if $\ell \gg \xi$, the force virtually vanishes at the critical composition.

The organization of this paper is as follows. In Sec.II, we will shortly explain the FPD method and the local functional theory applied to our problem. In Sec.III, we will explain the simulation method. In Sec.IV, we will present simulation results of bridging dynamics.

\section{THEORETICAL BACKGROUND}

\section{A. Critical behavior}

We consider a near-critical binary mixture with an upper critical solution temperature $T_{c}$ for small reduced temperature $\tau=T / T_{c}-1$ at a given pressure, where $T$ is above the wetting temperature $T_{\mathrm{w}}{ }^{17,33}$. (For mixtures with a lower critical solution temperature such as 2,6lutidine and water ( $\mathrm{LW})$, we should set $\tau=1-T / T_{c}$.) The order parameter $\psi$ is a scaled concentration deviation slightly deviating from its critical value. For $\tau<0$, a two-phase region appears in the $\tau-\psi$ plane. The coexistence curve (CX) consists of the favored and disfavored branches as

$$
\psi= \pm \psi_{\mathrm{cx}}= \pm b_{\mathrm{cx}}|\tau|^{\beta}
$$

where $b_{\text {cx }}$ is a constant. The critical exponents $54,63,64$ are given by $\beta \cong 0.325, \nu \cong 0.630$, and $\hat{\eta} \cong 0.032$. The correlation length $\xi$ depends on $\tau$ and $\psi^{2}$ and is given by $\xi_{0} \tau^{-\nu}$ for $\tau>0$ and $\psi=0$ and by $\xi_{0}^{\prime}|\tau|^{-\nu}$ for $\tau<0$ on CX, where $\nu$ is assumed to be common in the two cases and $\xi_{0}$ and $\xi_{0}^{\prime}$ are microscopic lengths. Here, $\xi_{0}$ is usually in the range $2-3 \AA$ for low molecular-weight binary mixtures. The ratio $\xi_{0} / \xi_{0}^{\prime}$ is a universal number in the renormal- ization group theory ${ }^{54}$ and its reliable estimate ${ }^{63}$ is 1.9 , so we suppose $\xi_{0}^{\prime} \sim 1 \AA$.

We express the free energy density $f(\psi, \tau)$ in accord with the scaling relations in the vicinity of the critical point in the $\tau-\psi$ plane including the region inside CX $\left(\tau<0,|\psi|<\psi_{\text {cx }}\right)$. This is needed because $\psi$ changes from positive to negative around the colloidal particles in the presence of strong adsorption. To this end, we use a simple parametric form of $f(\psi, \tau)$ devised in our previous paper ${ }^{47}$ (see the appendix).

At the starting point of our theory, the thermal fluctuations of $\psi$ and the velocity field $\boldsymbol{v}$ with wave numbers larger than $\xi^{-1}$ have already been coarse-grained or renormalized. Here, $\xi^{-1}$ is the lower cut-off wave number of the renormalization effect, so the free energy density $f(\psi, \tau)$ and the kinetic coefficients ( $\lambda$ in eqn (6) and $\eta_{0}$ in eqn (9)) are renormalized ones depending on fractional powers of $\xi^{-1}$. Then, since our dynamic equations have renormalized coefficients, they can well describe nonequilibrium processes with spatial scales longer than $\xi$ even without the thermal noise terms ${ }^{52}-54$. We also neglect Brownian motions of the colloidal particles.

\section{B. Strong adsorption near criticality}

As the critical point is approached, the strong adsorption regime is eventually realized outside CX due to a nonvanishing surface field $h_{1}$ (see eqn (4) and (5) $47,48,55,60-62$. Here, let $\psi$ be positive near the colloid surfaces. If the distance $z$ from such a wall is shorter than the bulk correlation length $\xi$ in the case $\xi \ll a, \psi(z)$ behaves as 47,60

$$
\psi \cong A_{0}\left(z+\ell_{0}\right)^{-\beta / \nu} \quad(0<z<\xi),
$$

where $A_{0}$ is a constant and $\ell_{0}$ is a microscopic length. The surface value of $\psi$ at $z=0$ satisfies $\psi_{0} \cong A_{0} \ell_{0}^{-\beta / \nu} \gg$ $\psi_{\mathrm{cx}}$ in the strong adsorption condition. Since $\beta / \nu \cong$ 0.52 , the preferential adsorption in the layer $z<\xi$ grows as $\int_{0}^{\xi} d z \psi \sim A_{0} \xi^{1-\beta / \nu}$ per unit area, resulting in the well-known critical adsorption, where the integral in the region $0<z<\ell_{0}$ is negligible and the strong adsorption limit is well-defined.

For $z>\xi, \psi$ depends on its value $\psi_{\infty}$ far from the wall. It decays as $\psi \sim A_{0} \xi^{-\beta / \nu} e^{-z / \xi}$ for $\psi_{\infty}=0$ at the critical composition, but it changes its sign from positive to negative for $\psi_{\infty}<0$. In this paper, we set $\psi_{\infty} \cong$ $-\psi_{\text {cx }}$ outside $\mathrm{CX}$, where the thickness of the transition layer is about $5 \xi$ with enlarged composition disturbances around the particles 47,48 . In accord with this result, a light scattering experiment ${ }^{17}$ showed that the adsorption layers of colloidal particles in LW were much thicker near the disfavored branch of CX than near the favored one.

In the literature ${ }^{9}-12,21,22,27-30,32$, the adsorptioninduced interaction has been called the critical Casimir interaction. However, the original (quantum) Casimir interaction stems from the ground-state fluctuations of the 
electromagnetic field between two mirrors ${ }^{65}$. In nearcritical fluids, more analogous is the interaction arising from the thermal fluctuations of $\psi$ at zero-surface field $h_{1}=0$, where the thermal average $\langle\psi\rangle$ is homogeneous. Notice that the fluctuation-induced interaction ${ }^{65}$ is much weaker than the adsorption-induced one (with inhomogeneous $\langle\psi\rangle)^{8,55}$. In fact, the Casimir amplitudes at $h_{1}=0$ (in the Neumann boundary condition) $)^{66}$ are much smaller than those under strong adsorption by one order of magnitude $\mathrm{e}^{-}-12,47,56,57$.

\section{Fluid-particle dynamics (FPD) near criticality}

In the FPD method $1,7,51,58,59$, the solid-liquid boundaries are treated as diffuse interfaces with thickness $d$ much shorter than the radius $a$, which much simplifies the calculation of colloid motions. We introduce the colloid shape function $\theta(\boldsymbol{r})=\sum_{k} \theta_{k}(\boldsymbol{r})(k=1,2$ here) with

$$
\theta_{k}(\boldsymbol{r})=\frac{1}{2}+\frac{1}{2} \tanh \left[\frac{1}{d}\left(a-\left|\boldsymbol{r}-\boldsymbol{R}_{k}\right|\right)\right],
$$

which tends to 1 in the colloid interior and to zero in the colloid exterior. We define $\psi$ even in the colloid interior and assume the total free energy $F$ of the form? $7,51,58$

$$
\begin{aligned}
F & =\int d \boldsymbol{r}\left\{(1-\theta)\left[f(\psi, \tau)+\frac{C}{2}|\nabla \psi|^{2}\right]+\theta \chi_{0}\left(\psi-\psi_{\mathrm{in}}\right)^{2}\right\} \\
& -\left(h_{1} / 3 d\right) \int d \boldsymbol{r} \psi \sum_{k}\left|\nabla \theta_{k}\right|^{2}+U\left(\boldsymbol{R}_{1}, \boldsymbol{R}_{2}\right),
\end{aligned}
$$

where the integration is within the cell. In the first line, $f(\psi, \tau)$ is the free energy density, $C$ is a weakly singular positive coefficient, and the term proportional to $\chi_{0}$ serves to fix $\psi$ at $\psi_{\text {in }}$ in the colloid interior. See the appendix for $f(\psi, \tau)$ and $C$. In the previous papers $7,51,58$, the mean field form of $f$ has been used. In the second line, $h_{1}$ is the surface field assuming a large positive number $33,47,48$ and $U\left(\boldsymbol{R}_{1}, \boldsymbol{R}_{2}\right)$ is an applied potential acting on the particles. In the limit $d / a \rightarrow 0$, the second term tends to the usual surface integral $-h_{1} \int d S \psi$ and use of eqn (2) yields

$$
h_{1} \cong(\beta / \nu) C \psi_{0} / \ell_{0},
$$

where $\psi_{0}$ is the surface value of $\psi$. In the strong adsorption condition $\psi_{0} \gg \psi_{\mathrm{cx}}$, we find $h_{1} \propto \psi_{0}^{2 \nu / \beta-1}$ from $C \propto \psi_{0}^{-\hat{\eta} \nu / \beta}$ (see the appendix).

Assuming a homogeneous $\tau$, we use the model $\mathrm{H}$ dynamics $52-54$. The order parameter $\psi$ is governed by

$$
\frac{\partial}{\partial t} \psi=-\nabla \cdot[\psi \boldsymbol{v}-\lambda \nabla \mu],
$$

where $\boldsymbol{v}$ is the velocity field assumed to be incompressible $(\nabla \cdot \boldsymbol{v}=0), \lambda$ is the kinetic coefficient, and $\mu=\delta F / \delta \psi$ is the (generalized) chemical potential. The particle velocities are given by the average interior velocity field,

$$
\frac{d}{d t} \boldsymbol{R}_{k}=\frac{1}{v_{0}} \int d \boldsymbol{r} \theta_{k} \boldsymbol{v}
$$

where $v_{0}=\int d \boldsymbol{r} \theta_{k} \cong 4 \pi a^{3} / 3$. Neglecting the acceleration (the Stokes approximation), we determine $\boldsymbol{v}$ from

$$
\psi \nabla \mu+\frac{1}{v_{0}} \sum_{k} \theta_{k} \mathcal{F}_{k}+\nabla p_{1}=\nabla \cdot \stackrel{\leftrightarrow}{\sigma}_{\mathrm{vis}}
$$

where $\mathcal{F}_{k}=\partial F / \partial \boldsymbol{R}_{k}, p_{1}$ is a pressure ensuring $\nabla \cdot \boldsymbol{v}=0$, and $\overleftrightarrow{\sigma}_{\text {vis }}=\left\{\sigma_{i j}\right\}$ is the viscous stress tensor of the form,

$$
\sigma_{i j}=\left(\eta_{0}+\eta_{1} \theta\right)\left(\nabla_{j} v_{i}+\nabla_{i} v_{j}\right),
$$

with $\nabla_{i}=\partial / \partial x_{i}(i=x, y, z)$. In the FPD method, the colloidal particles are treated as a highly viscous fluid. Then, $\eta_{0}$ is the viscosity in the liquid and $\eta_{0}+\eta_{1}$ is the viscosity in the colloid interior with $\eta_{1} \gg \eta_{0}$. If we use eqn (6)-(9), $F$ in eqn (4) monotonically decreases in time $(d F / d t \leq 0)$ in nonequilibrium ${ }^{54}$.

The kinetic coefficient $\lambda$ in eqn (6) is strongly enhanced due to the convective motions of the critical fluctuations ${ }^{52-54}$. Because the solvent is close to CX in our situation, we set $\lambda$ equal to its value on $\mathrm{CX}$ as

$$
\lambda=\chi_{\mathrm{cx}} D / k_{B} T_{c},
$$

where $\chi_{\mathrm{cx}}$ is the susceptibility on CX in eqn (A1) and $D$ is the diffusion constant in the Stokes form,

$$
D=k_{B} T_{c} / 6 \pi \eta_{0} \xi
$$

with $\xi=\xi_{0}^{\prime}|\tau|^{-\nu}$. For $\xi \gg \xi_{0}$, this $D$ is much smaller than microscopic diffusion constants. In contrast, the viscosity $\eta_{0}$ in eqn (9) is only weakly singular and may be treated as a constant independent of $\tau$ and $\psi$ in nearcritical fluids.

\section{SIMULATION BACKGROUND}

In the presence of colloidal particles with radius $a$, we define characteristic values of $\tau$ and $\psi$ by $\underline{\underline{48}}$

$$
\tau_{a}=\left(\xi_{0} / a\right)^{1 / \nu}, \quad \psi_{a}=b^{\prime} \tau_{a}^{\beta},
$$

where the ratio $b^{\prime} / b_{\mathrm{cx}}$ is calculated to be 1.47 in our scheme ${ }^{47}$. We consider negative homogeneous $\tau$ with considerably large $|\tau| / \tau_{a}=\left(\xi_{0}^{\prime} a / \xi_{0} \xi\right)^{1 / \nu} \gg 1$, so we treat the case $\xi \ll a$. Hereafter, we measure time $t$ in our simulation in units of $t_{0}$ defined by

$$
t_{0}=a^{2} / D=(a / \xi)^{2} t_{\xi},
$$

where $t_{\xi}=6 \pi \eta_{0} \xi^{3} / k_{B} T$ is the thermal relaxation time of the critical fluctuations 52,54 . As the parameters in eqn (3) and (4), we set $d=a / 8, \chi_{0}=783 k_{B} T / a^{3} \psi_{a}^{2}$, $\psi_{\text {in }}=5 \psi_{a}$, and $h_{1} / 3 d=15.7 k_{B} T / a \psi_{a}$. The viscosity ratio is $\eta_{1} / \eta_{0}=100$.

Let us assume $\tau=-8 \tau_{a}$ for a LW mixture slightly outside CX, for which $T_{c}=307 \mathrm{~K}, \xi_{0}=2.5 \AA$, and $\eta_{0}=$ $2.0 \mathrm{cp}$. Then, for $a=200 \mathrm{~nm}$, we obtain $\tau_{a}=1.8 \times 10^{-5}$, $\tau=-1.5 \times 10^{-4}, \xi=29 \mathrm{~nm}, D=3.5 \times 10^{-8} \mathrm{~cm}^{2} / \mathrm{s}$, 


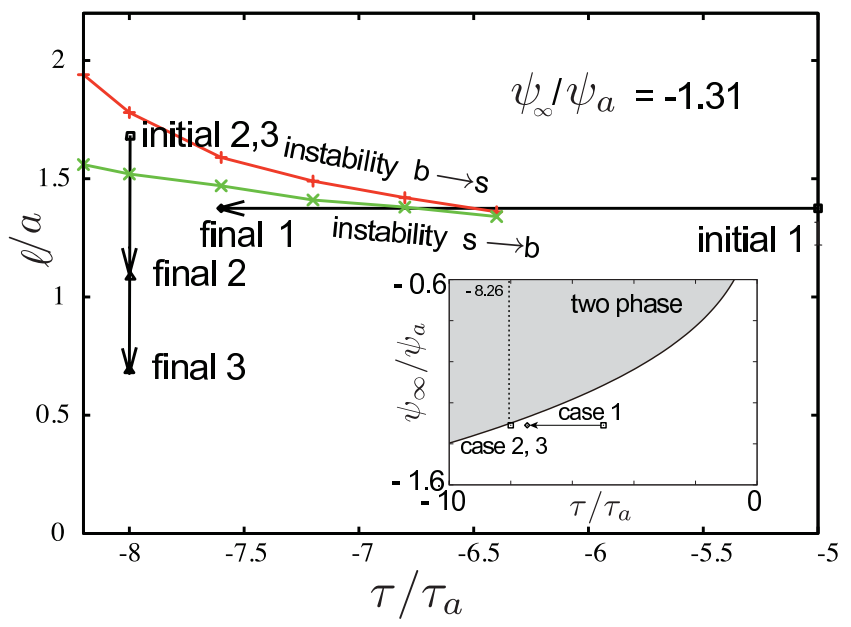

FIG. 1: Illustration of simulation from separated to bridged states of two particles outside CX in the $\tau / \tau_{a}-\ell / a$ plane, where $\psi$ tends to $\psi_{\infty} \cong-1.31 \psi_{a}$ far from the particles. If $\psi=-1.31 \psi_{a}$, the reduced temperature on $\mathrm{CX}$ is given by $\tau_{\mathrm{cx}}=-8.26 \tau_{a}$ (vertical dotted line). In case $1, \tau / \tau_{a}$ is lowered from -5.0 to -7.6 at nearly fixed separation $\ell=1.38$. In cases 2 and $3, \ell / a$ changes from 1.69 to 1.09 and 0.69 , respectively, at $\tau / \tau_{a}=-8.0$. Below $(\mathrm{s} \rightarrow \mathrm{b}$ ) instability line (lower green line) separated states become unstable, while above (b $\rightarrow$ s) instability line (upper red line) bridged states become unstable. In inset, these three cases are illustrated in the $\tau / \tau_{a}-\psi_{\infty} / \psi_{a}$ plane outside CX or below the two-phase region (in gray).

$t_{\xi}=2.4 \times 10^{-4} \mathrm{~s}$, and $t_{0}=12 \mathrm{~ms}$. If we increase $a$ to 1 $\mu \mathrm{m}, t_{0}$ becomes $1.4 \mathrm{~s}$.

We place two particles in the middle of a cylindrical cell with radius $L_{0}=3.75 a$ and height $H_{0}=10 a$, so the system is in the region $\rho=\left(x^{2}+y^{2}\right)^{1 / 2}<L_{0}$ and $-H_{0} / 2<z<H_{0} / 2$. The particle centers are at $(0,0, \pm(\ell / 2+a))$, where $\ell$ is the surface-to-surface separation distance. This geometry is axisymmetric, so the time integration was performed in the $2 \mathrm{D} \rho-z$ plane, where the mesh size is $\Delta x=a / 16$ and the time interval width is $\Delta t=5 \times 10^{-6} t_{0}$. The periodic boundary condition is imposed along the $z$ axis, while we set $\partial \psi / \partial \rho=0$ and $\boldsymbol{v}=\mathbf{0}$ on the side wall $\rho=L_{0}$. Then, the total order parameter $\int d \boldsymbol{r} \psi$ is conserved in time. As a result, the value of $\psi$ away from the particles, written as $\psi_{\infty}$, exhibits a slight decrease of order $0.02 \psi_{a}$ after bridging. We also find that $\left|\psi-\psi_{\text {in }}\right|$ in the colloid interior remains smaller than $0.01 \psi_{a}$ for our choice of $\chi_{0}$ and $\psi_{\text {in }}$, which assures the validity of our model.

Even in the axisymmetric geometry, integration of eqs (6) and (7) under eqn (8) is time-consuming. However, if we fix the particle positions, we may efficiently seek the equilibrium profiles of $\psi$ from the relaxation equation ${ }^{49}$,

$$
\frac{\partial}{\partial t} \psi=-\mu+\mu_{\infty}
$$

where $\mu=\delta F / \delta \psi$ with $\mu_{\infty}$ being its value far from the particles. Here, $\boldsymbol{v}$ is not coupled. At long times, the stationary solution satisfies $\mu=\mu_{\infty}$.

\section{BRIDGING AND AGGREGATION DYNAMICS}

\section{A. Situations of bridging}

In Fig.1, we illustrate how we performed our simulation outside CX. We initially set $\psi_{\infty}=-1.31 \psi_{a}$ far from the particles. For this concentration, the reduced temperature on CX is given by $\tau_{\mathrm{cx}}=-8.26 \tau_{a}$ from eqn (1), so we set $\tau>\tau_{\text {cx }}$. We plot the instability line from separated to bridged states $(\mathrm{s} \rightarrow \mathrm{b})$ and that from bridged to separated states $(\mathrm{b} \rightarrow \mathrm{s})$. To determine these two instability lines, we integrated the relaxation equation (14) at fixed particle positions for various $\ell$ and $\tau$. We detected growth of small disturbances when we crossed the former (latter) line by gradually decreasing (increasing) $\ell$ from separated (bridged) states. Between these lines, both separated and bridged states remained stationary. Furthermore, between them, there is a first-order transition line, on which the free energy assumes the same value for these two $\operatorname{states}^{48}$.

In our simulation, even if $\ell \gg \xi$, the adsorptioninduced force is significant from the beginning. Here, it is long-ranged, decaying as $\propto e^{-\ell / \xi}$ only for $\ell \gtrsim a$, due to the expanded adsorption layers close to the disfavored branch of CX (see Sec.IIA). See such examples in Fig.5 in our paper ${ }^{48}$. Indeed, if the initial attractive force is equated to $k_{B} T A a \xi^{-2} e^{-\ell / \xi}$ for case 1 in Fig.1, we have $A=449$, while $A \sim 4$ at the critical composition 27.48 .

In real experiments, charges usually appear on the colloid surfaces in aqueous mixtures ${ }^{14,15,18-22,32}$. Supposing weak ionization, we neglect the effect of charges on the adsorption-induced interaction. However, we will assume a charge-induced repulsive interaction effective at short separation (in eqn (19) below ) ${ }^{31}$. We also neglect the van der Waals interaction ${ }^{31,33}$ in our simulation. We note that Bonn et al. ${ }^{22}$ observed colloid aggregation in refractive-index-matched systems, where the van der Waals interaction was suppressed.

\section{B. Bridging after temperature quenching at fixed $\ell$}

In case 1 simulation, at $t=0$, we lowered $\tau$ from $-5 \tau_{a}$ $(t \leq 0)$ to $-7.6 \tau_{a}(t>0)$ across the $(\mathrm{s} \rightarrow \mathrm{b})$ instability line, where $\psi_{\infty} \cong-1.31 \psi_{a}$ outside CX. For $t<0$, we realized the equilibrium at fixed particle positions as a stationary solution of eqn (14). The correlation length $\xi$ in the bulk is $0.09 a$ in the final state. Supposing optical tweezers ${ }^{67.68}$, we choose the potential $U$ in eqn (4) as

$$
U=\frac{1}{2} K \sum_{k=1,2}\left(Z_{k}-Z_{k}^{0}\right)^{2},
$$

where $Z_{1}$ and $Z_{2}$ are the $z$ coordinates of the particle centers and $Z_{1}^{0}$ and $Z_{2}^{0}$ are their initial values. The spring 
(a) Case 1 initial: $\tau / \tau_{a}=-5$

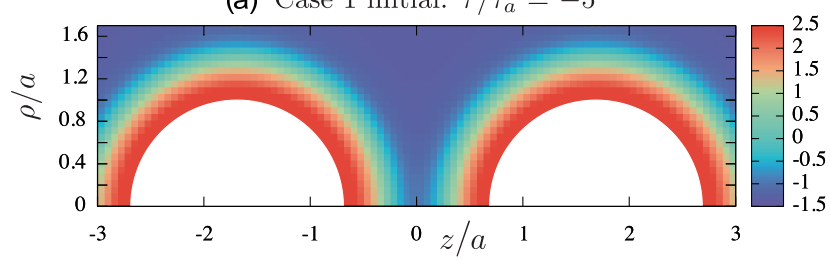

(b) Case 1 final: $\tau / \tau_{a}=-7.6$

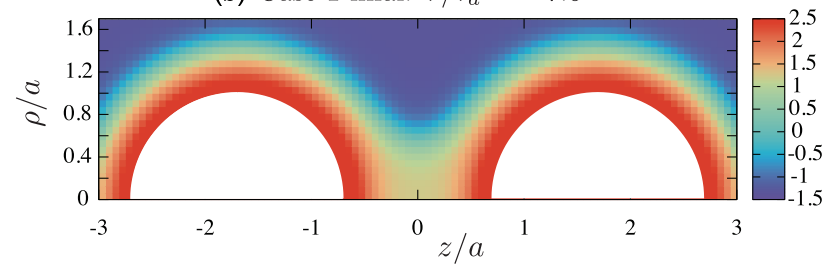

FIG. 2: Profiles of $\psi(\rho, z, t) / \psi_{a}$ in (a) the initial state and (b) the final state in the $\rho$ - $z$ plane (in gradation) in case 1 , where $\tau / \tau_{a}$ is lowered from -5 to -7.6 at fixed $\psi_{\infty}$ and $\ell$ in strong adsorption near criticality.
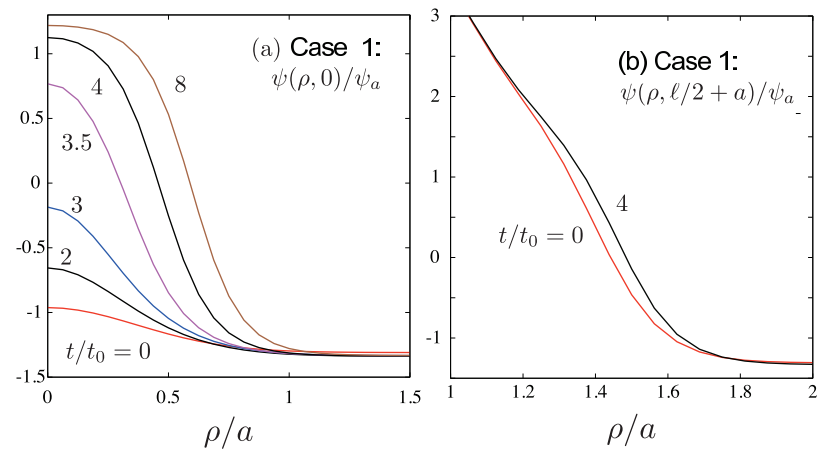

FIG. 3: Time evolution of $\psi(\rho, z, t) / \psi_{a}$ vs $\rho / a$ in case 1 . (a) Profiles on the midplane $(z=0)$ at $t / t_{0}=0,2,3,3.5,4$, and 8, where a bridging domain appears. (b) Those on the plane containing the particle center $(z=\ell / 2+a)$ at $t / t_{0}=0$ and 4 , where the adsorption layer is nearly stationary.
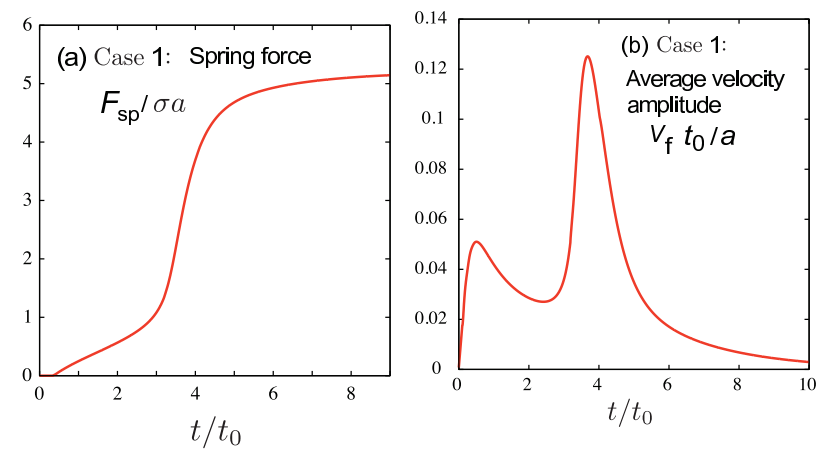

FIG. 4: (a) Spring force $F_{\mathrm{sp}}(t)$ in eqn (16) divided by $\sigma a$ vs $t / t_{0}$, which increases abruptly with bridging. (b) Averaged velocity amplitude $v_{\mathrm{f}}(t)$ in eqn (18) divided by $a / t_{0}=D / a$ vs $t / t_{0}$, exhibiting two peaks at $t / t_{0}=0.5$ and 3.7 . Instability growth is slow initially $\left(t / t_{0}<2\right)$ and is accelerated on bridge formation $\left(t / t_{0} \sim 4\right)$. constant $K$ is set equal to $5300 k_{B} T / a^{2}$, which depends on $a$ and is $220 \mathrm{pN} / \mu \mathrm{m}$ for $a=1 \mu \mathrm{m}$. Then, the displacement $Z_{1}^{0}-Z_{1}$ is $0.0085 a$ after bridging, where the separation $\ell$ is shortened from its initial length $1.38 a$ by $1.2 \%$. The time-dependent spring force is given by

$$
F_{\mathrm{sp}}(t)=K\left[Z_{1}^{0}-Z_{1}(t)\right]=K\left[Z_{2}(t)-Z_{2}^{0}\right],
$$

which is balanced with the force on the particles from the fluid in the Stokes approximation ${ }^{48}$.

In Fig.2, we give the initial and final profiles of $\psi(\rho, z)$ in gradation. We can see thick adsorption layers covering the colloid surfaces due to large $h_{1}$ in eqn (4) and (5) and a bridging region with $\psi \cong \psi_{\mathrm{cx}}$ between the particles in the final state. In contrast, if the system would be quenched slightly inside CX, thick wetting layers with sharp interfaces would cover the whole particle surfaces?

In Fig.3, we display time evolution of $\psi(\rho, z, t)$ vs $\rho$ on (a) the midplane $(z=0)$ and (b) the plane containing the upper particle center $(z=\ell / 2+a)$ far from the other particle. In $(\mathrm{a}), \psi(\rho, 0, t)$ increases between the particles. Its growth is rapid in the time range $3<t / t_{0}<4$. For $t>4$, it exhibits a flat profile in the middle region $\rho / a \lesssim 0.2$ and an interface becomes well-defined. In (b), $\psi(\rho, \ell / 2+a, t)$ exhibits strong adsorption behavior, which only slightly depends on $t$.

In Fig.4(a), we plot the spring force $F_{\mathrm{sp}}(t)$ in eqn (16). It increases rather slowly for $t / t_{0}<3$, but abruptly for $3<t / t_{0}<4$ up to the capillary force $41,44,48$,

$$
F_{\mathrm{sp}} \sim 2 \pi \sigma a \sim k_{B} T a / \xi^{2}
$$

where $\sigma$ is the surface tension in eqn (A3). In Sec.I, the capillary force has already been discussed. See the appendix of our previous paper for its more systematic derivation $^{48}$. This sharp increase of $F_{\mathrm{sp}}(t)$ is simultaneous with the interface formation (see Fig.3(a)). Remarkably, $F_{\mathrm{sp}}(t)$ becomes of order $\sigma a \sim 0.1 k_{B} T a / \xi^{2}$ before the bridging transition $\left(t<3 t_{0}\right)$. As noted at the beginning of this section, the adsorption-induced force is long-ranged in pretransitional states close to the bridging transition.

We are interested in the fluid velocity outside the particles. In Fig.4(b), we thus show the average velocity amplitude $v_{\mathrm{f}}(t)$ in the fluid region defined by

$$
v_{\mathrm{f}}(t)^{2}=\int d \boldsymbol{r}(1-\theta) v^{2} /\left(4 \pi a^{3} / 3\right),
$$

where $1-\theta$ tends to 1 outside the particles. We can see that $v_{\mathrm{f}}(t)$ has a small peak at $t / t_{0}=0.5$, a minimum at $t / t_{0}=2.4$, and a large sharp peak at $t / t_{0}=3.7$. In the initial stage $t / t_{0}<0.5$, the adsorption layers of the two particles weakly merge without domain formation, causing a weak convective flow. This initial motion decays for $t / t_{0}>0.5$. However, an unstable mode emerges giving rise to an increase in $\psi$ from the center line $\rho=0$. It is slowly growing and not noticeable for $t / t_{0}<2$, but it grows abruptly in the late stage $t / t_{0}>3$ with outward expansion of an interface from the center line. This 
(a) $\boldsymbol{V}$ Case1: $t / t_{0}=4$

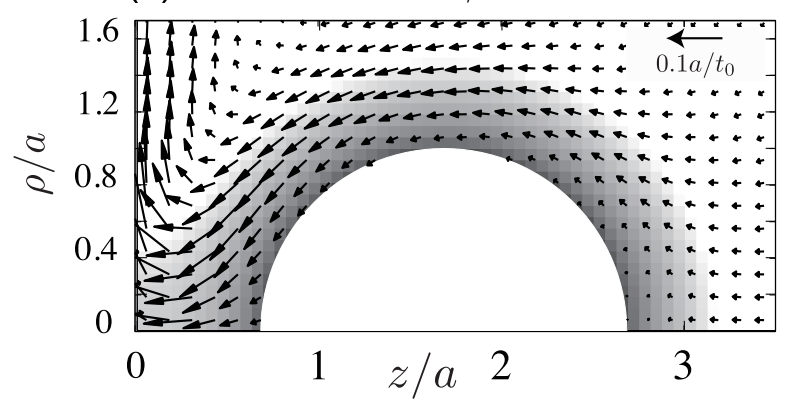

(b) $\psi \boldsymbol{v}-\lambda \nabla \mu \quad$ Case1: $t / t_{0}=4$

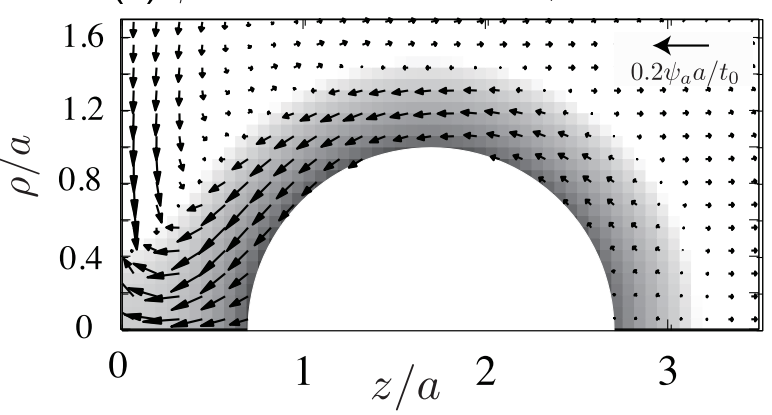

FIG. 5: (a) Velocity field $\boldsymbol{v}$ and (b) concentration flux $\psi \boldsymbol{v}-\lambda \nabla \mu$ at $t / t_{0}=4.0$ in case 1 . Arrow lengths are written according to standard lengths (a) $0.1 a / t_{0}$ and (b) $0.2 \psi_{a} a / t_{0}$. Flow is significant around the particle in the adsorption region $\psi>0$ (in gray) and close to the midplane.
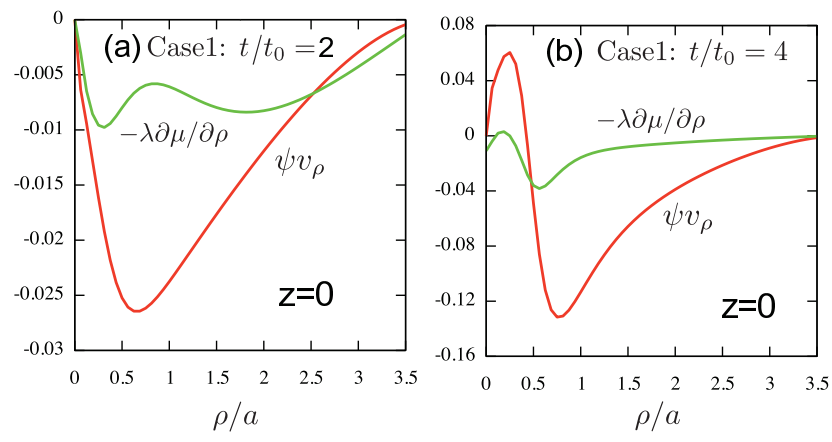

FIG. 6: Convective part $\psi v_{\rho}$ and diffusive part $-\lambda \partial \mu / \partial \rho$ in the concentration flux vs $\rho / a$ in the midplane $(z=0)$ at (a) $t / t_{0}=2$ and (b) 4 in case 1 . The former is mostly larger than the latter by one order of magnitude. The vertical axes are written in units of $\psi_{a} a / t_{0}=D \psi_{a} / a$.

final acceleration of the growth is obviously due to the hydrodynamic transport.

In Fig.5, we display the profiles of the velocity field $\boldsymbol{v}$ in (a) and the concentration flux $\psi \boldsymbol{v}-\lambda \nabla \mu$ in (b) at $t / t_{0}=4$. Here, the vectors $\boldsymbol{v}$ and $\nabla \mu$ are expressed as $\boldsymbol{v}=v_{\rho} \boldsymbol{e}_{\rho}+v_{z} \boldsymbol{e}_{z}$ and $\nabla \mu=(\partial \mu / \partial \rho) \boldsymbol{e}_{\rho}+(\partial \mu / \partial z) \boldsymbol{e}_{z}$, where $\boldsymbol{e}_{\rho}=(x / \rho, y / \rho, 0)$ and $\boldsymbol{e}_{z}=(0,0,1)$. At $t / t_{0}=4$, $v_{\mathrm{f}}$ is large in Fig.4(b). The maximum velocity is of order $0.1 a / t_{0}=0.1 D / a$. We can see that $\boldsymbol{v}$ (nearly) van- (a) Cases 2 and 3 initial: $\ell / a=1.69$

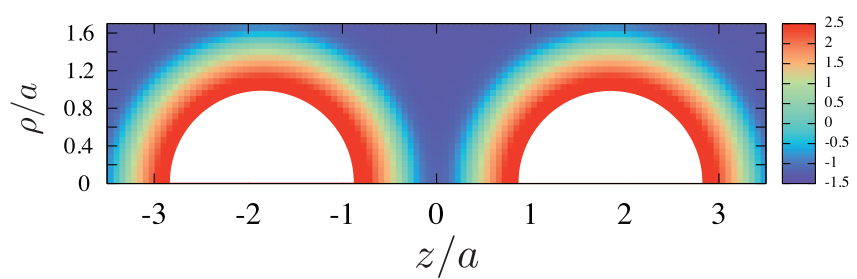

(b) Case 2 final: $\ell / a=1.09$

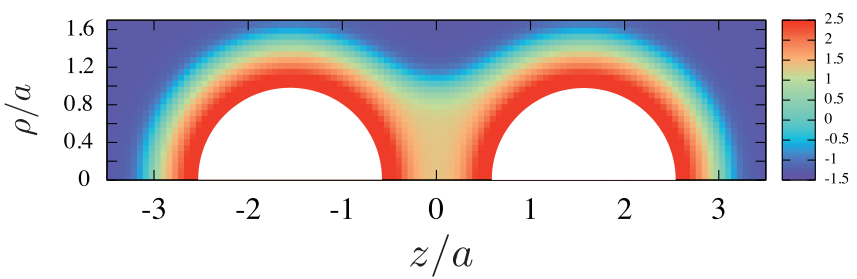

(c) Case 3 final: $\ell / a=0.69$

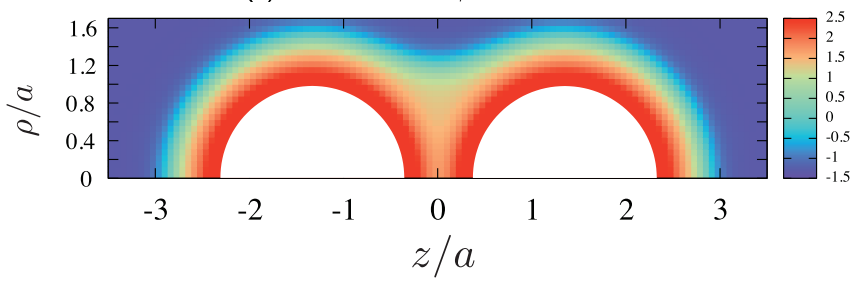

FIG. 7: Profiles of $\psi(\rho, z) / \psi_{a}$ in the $\rho$ - $z$ plane (in gradation) with $\tau / \tau_{a}=-8$ and $\psi_{\infty}=-1.31 \psi_{a}$ in strong adsorption. (a) The initial state for cases 2 and 3 , where $\ell / a=1.69$. (b) The final state in case 2 , where $\ell / a=1.09$. (c) The final state in case 3 , where $\ell / a=0.69$.

ishes on the colloid surface, while $\psi \boldsymbol{v}-\lambda \nabla \mu$ is significant within the adsorption layer with $\psi>0$. The flow is then directed outward on the midplane due to the incompressibility condition $\nabla \cdot \boldsymbol{v}=0$. This squeezing flow is blocked at the side wall at $\rho=3.75 a$, but the flow in our case is appreciable only for a short time interval and the boundary disturbance should little affect the bridging dynamics. For LW, the typical velocity is estimated as $0.1 a / t_{0}=1.7 \times 10^{-4} \mathrm{~cm} / \mathrm{s}$ for $a=200 \mathrm{~nm}$.

In Fig.6, we give the $\rho$-components of the convective flux and the diffusive flux, $\psi v_{\rho}$ and $-\lambda \partial \mu / \partial \rho$, separately, on the midplane $z=0$ at $t / t_{0}=2$ in (a) and 4 in (b). The velocity amplitude is one order of magnitude larger in (b) than in (a). Squeezing can be seen in the region $\rho / a<$ 0.5 in (b). We recognize that $\psi v_{\rho}$ is mostly larger than $-\lambda \nabla_{\rho} \mu$ by one order of magnitude, which demonstrates relevance of the hydrodynamic flow in bridging.

In their simulation, Araki and Tanaka ${ }^{7}$ pinned two particles in a binary mixture and quenched their system inside CX $\left(\psi_{\infty}=-0.8 \psi_{\mathrm{cx}}\right)$ to realize formation of wetting layers around the particles. They found that the spring force increased above the capillary force transiently in an initial stage for small separation distances. This effect 

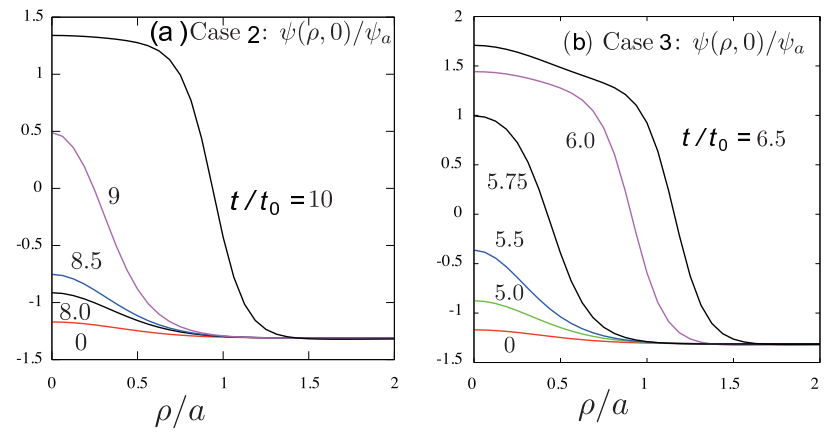

FIG. 8: Time evolution of $\psi(\rho, 0, t) / \psi_{a}$ on the midplane $z=0$ vs $\rho / a$, where (a) $t / t_{0}=0,8,8.5,9$, and 10 in case 2 and (b) $t / t_{0}=0,5,5.5,5.75,6$, and 6.5 in case 3 . In (a), a well-defined bridging domain appears with a flat profile in the time range $9<t / t_{0}<10$. In (b), $\psi(\rho, 0, t)$ increases abruptly in the time range $5<t / t_{0}<6$, where the adsorption layers of the two particles merge.
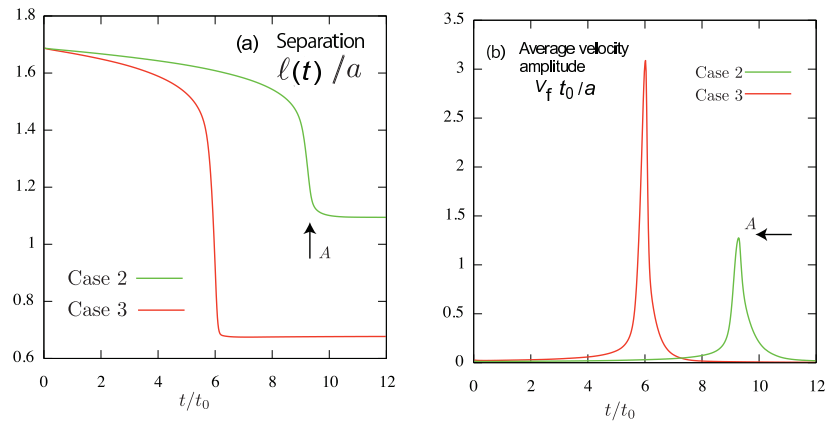

FIG. 9: (a) Separation distance $\ell(t)$ divided by $a$ vs $t / t_{0}$, where the maximum of $|d \ell(t) / d t|$ is $0.457 a / t_{0}$ at $t=9.24 t_{0}$ in case 2 and $1.27 a / t_{0}$ at $t=6.00 t_{0}$ in case 3 . The initial separation $\ell(0)$ is about $11 \xi$. (b) Averaged velocity amplitude $v_{\mathrm{f}}$ in eqn (18) divided by $a / t_{0}$ vs $t / t_{0}$ in cases 2 and 3 . Peaks are at squeezing.

has not yet been understood.

\section{Bridging between aggregating two particles}

In cases 2 and 3 , we let the two particles approach from the initial separation $\ell(0)=1.69 a$ for $t>0$, where we set $\tau / \tau_{a}=-8$ and $\psi_{\infty}=-1.31 \psi_{a}$. Then, we have $\ell(0) / \xi \sim 11$. For $t<0$, the particle positions were fixed and the fluid was in equilibrium, which was attained as a stationary solution of eqn (14). For $t>0$, the motions are caused by the adsorption-mediated attractive interaction. Notice that the initial state is located slightly below the $\mathrm{b} \rightarrow \mathrm{s}$ instability line in Fig.1. This is because the initial particle motions become extremely slow above the $\mathrm{b} \rightarrow \mathrm{s}$ line in the present case.

The potential $U$ in eqn (4) is repulsive, preventing the particles from touching each other. We assume that it depends on $\ell=Z_{1}-Z_{2}-2 a$ exponentially as

$$
U=k_{B} T_{c} B e^{-\kappa \ell},
$$

where $B=4 \times 10^{5}$ in case 2 and $B=8 \times 10^{3}$ in case 3 with $\kappa=10 / a$. As a result, we have the final separation distance is $\ell(\infty)=1.09 a$ in case 2 and $0.69 a$ in case 3 . The above potential can arise for charged colloidal particles when the van der Waals interaction is negligible ${ }^{22}$. For weakly charged colloid particles, the screened Coulomb interaction in the Derjaguin, Landau, Verway, and Overbeek (DLVO) theory ${ }^{31}$ is of the form,

$$
U_{\mathrm{DLVO}}=\bar{Q}^{2} e^{-\kappa \ell} /\left[\varepsilon(1+\kappa a)^{2}(\ell+2 a)\right],
$$

where $\bar{Q}$ is the charge on a colloid particles, $\epsilon$ is the dielectric constant, and $\kappa$ is the Debye wave number. If $\kappa a \gg 1$ and $\ell+2 a \cong 2 a, U_{\text {DLVO }}$ assumes the form in eqn (19) with

$$
B=\bar{Q}^{2} /\left[2 k_{B} T_{c} \epsilon \kappa^{2} a^{3}\right]=8\left(\pi \rho_{s} / e\right)^{2} \ell_{B} a / \kappa^{2},
$$

where $\rho_{s}$ is the surface charge density and $\ell_{B}=e^{2} / k_{B} T_{c} \varepsilon$ is the Bjerrum length. For example ${ }^{22,27}$, we may set $2 \pi \rho_{s} / e \sim 1 / \mathrm{nm}^{2}$ and $\ell_{B} \sim 1 \mathrm{~nm}$ to obtain $B \sim 2 a^{3} /(a \kappa)^{2}$ with $a$ in nm, so the adopted values of $B$ in this paper can be realized in experiments.

In Fig.7, we give the profiles of $\psi(r, z) / \psi_{a}$ in the initial and final states in cases 2 and 3 . where the particles are bridged in the final state. The adsorption layers outside the bridged region $(|z|>\ell / 2)$ are nearly stationary as in Fig.2. In the final state in case 3 , the adsorption layers of the two particles are not well separated. In Fig.8, we show $\psi(\rho, 0, t) / \psi_{a}$ on the midplane vs $\rho / a$ at several times. In case $2, \psi(\rho, 0, t)$ becomes fairly flat with a welldefined interface in the middle $\rho / a<0.7$ for $t / t_{0}=10$. In case 3 , it depends on $\rho$ considerably even at the center for $t / t_{0}=8.5$. The $\psi$ between the particles increases abruptly in the time range $8.5<t / t_{0}<10$ in case 2 and $5.5<t / t_{0}<6.5$ in case 3 .

In Fig.9, we show the separation distance $\ell(t)$ and the average velocity amplitude $v_{\mathrm{f}}(t)$ in eqn (18). Here, the maximum of $|d \ell(t) / d t|$ is $0.457 a / t_{0}$ at $t=9.24 t_{0}$ in case 2 and is $1.27 a / t_{0}$ at $t=6.0 t_{0}$ in case 3 . The final approach in case 3 is very fast, where the fluid between the particles is rapidly squeezed out. In Fig.10, we display (a) $\boldsymbol{v}$, (b) $\boldsymbol{v}-\boldsymbol{v}_{c}$, and (c) $-\lambda \vec{\nabla} \mu+\psi \boldsymbol{v}$ at $t / t_{0}=9.28$, where $\boldsymbol{v}_{c}=-0.448\left(a / t_{0}\right) \boldsymbol{e}_{z}$ is the particle velocity with $\boldsymbol{e}_{z}$ being the unit vector along the $z$ axis. The maximum velocity is of order $a / t_{0}=D / a$, which is ten times larger than in Fig.5(a). Here, for LW, we have $a / t_{0}=1.7 \times 10^{-3}$ $\mathrm{cm} / \mathrm{s}$ for $a=200 \mathrm{~nm}$. In (b), the adsorption layer moves with the particle and squeezing takes place between the particles. In (c), the convective transport within the adsorption layer is crucial. In Fig.11, we compare $\psi v_{\rho}$ and $-\lambda \nabla_{\rho} \mu$ as in Fig.6, which demonstrates that the convective transport dominates over the diffusive transport.

It is rather surprising that the adsorption layer moves with the particle without large deformations in Fig.10(b), 

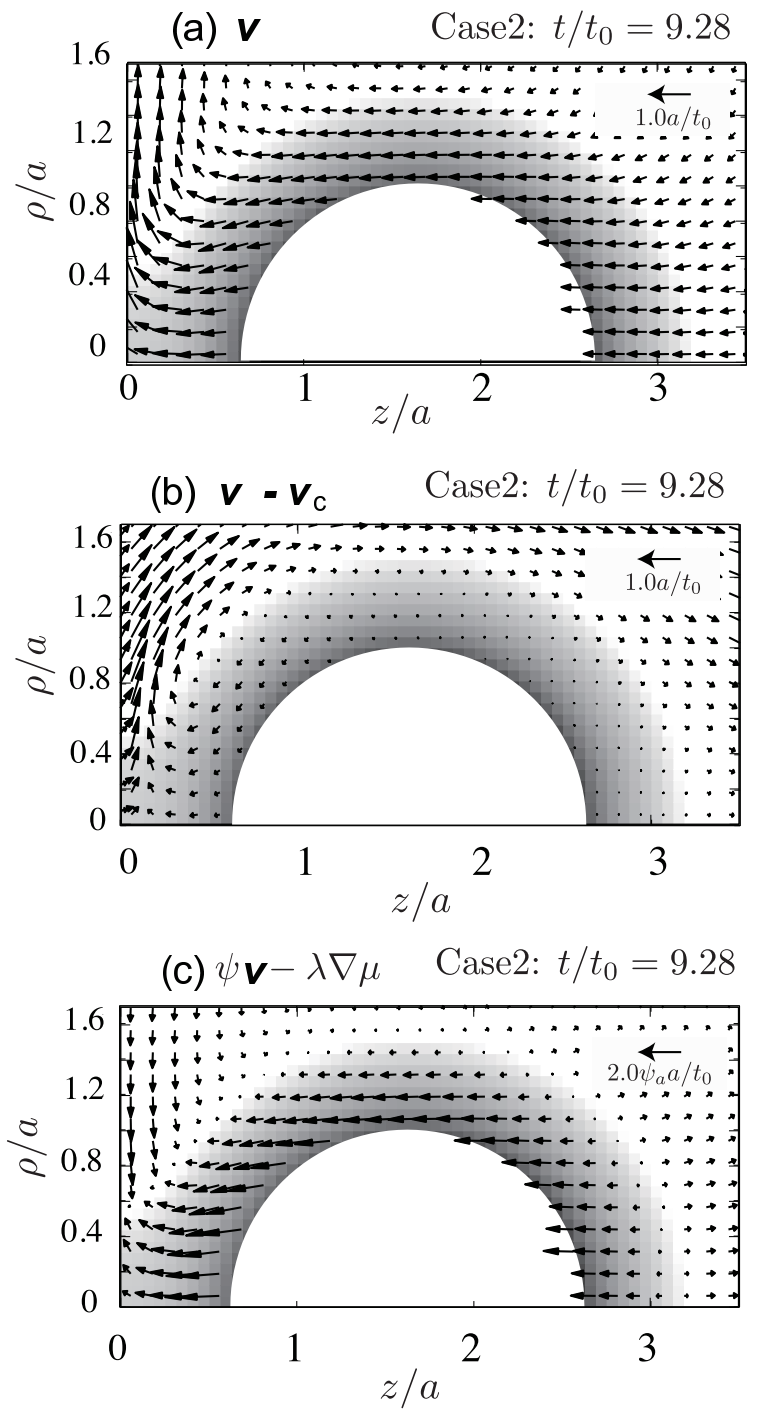

FIG. 10: (a) Velocity field $\boldsymbol{v}$, (b) $\boldsymbol{v}-\boldsymbol{v}_{c}$, and (c) concentration flux $\psi \boldsymbol{v}-\lambda \nabla \mu$ at $t / t_{0}=9.28$ in case 2 (point A in Fig.9), where $\boldsymbol{v}_{c}=-0.448\left(a / t_{0}\right) \boldsymbol{e}_{z}$ is the particle velocity. Arrow lengths are written according to standard lengths given by $1.0 a / t_{0}$ in (a) and (b) and $2.0 \psi_{a} a / t_{0}$ in (c). In (b), the adsorption layer with $\psi>0$ (in gray) moves with the particle far from the midplane.

though there are no sharp interfaces (see Fig.3(b)). This aspect should be further examined, which should give rise to a decrease in the diffusion constant of suspended particles even due to critical adsorption.

\section{SUMMARY AND REMARKS}

We have numerically examined dynamics of bridging and aggregation of two colloidal particles in a nearcritical binary mixture outside CX. Particularly near the disfavored branch of CX, the critical adsorption is strong and extended so that the adsorption-induced interaction is much enhanced. As a result, the bridging transition
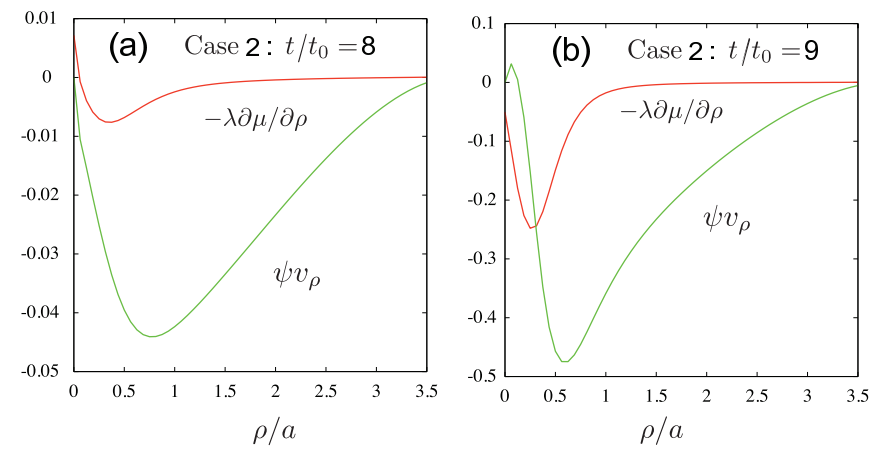

FIG. 11: Convective flux $\psi v_{\rho}$ and diffusive flux $-\lambda \partial \mu / \partial \rho$ vs $\rho / a$ in the midplane $(z=0)$ at (a) $t / t_{0}=8$ and (b) 9 in case 2. Comparison indicates that the hydrodynamic convection dominates over the diffusive transport. The vertical axes are written in units of $\psi_{a} a / t_{0}$.

readily takes place among the colloidal particles when the face-to-face separation $\ell$ is of the order the particle radius $a$. We have assumed that $a$ is much longer than the correlation length $\xi$. We summarize our main results in the following.

In Sec.II, we have explained the critical behavior, the FPD method, and the model $\mathrm{H}$ equations in the Ginzburg-Landau scheme. In Sec.III, we have explained the simulation method and introduced characteristic order parameter $\psi_{a}$, reduced temperature $\tau_{a}$, and time $t_{0}$.

In Sec.IV, to induce a bridging transition in a separated state, we have crossed the instability line in the $\tau-\ell$ plane by temperature quenching (case 1) and by two-particle aggregation (cases 2 and 3 ). In case 1 , the spring force $F_{\mathrm{sp}}(t)$ has been calculated as in Fig.4(a), which increases abruptly up to order $2 \pi \sigma a$ upon formation of a well-defined interface of a bridging domain. In cases 2 and 3 , the separation $\ell(t)$ has been obtained as in Fig.9(a), where the two particles initially approach due to the adsorption-induced attraction but eventually stop due to the screened Coulomb interaction in eqn (19). In case 2 , the final distance $\ell(\infty)$ is large and a well-defined bridging domain appears. In case 3, it is not large such that the adsorption layers of the two particles overlap. In the final stage in these cases, the fluid between the particles is squeezed out, leading to peaks in the average velocity amplitude $v_{\mathrm{f}}$ in Fig.9(b). In all these cases, the convective transport of $\psi$ within the adsorption layers is crucial.

Finally, we further mention two experiments, which suggest the presence of bridging in aggregation.

(1) Broide et al $\underline{\underline{16}}$ studied aggregation kinetics after a temperature change at $t=0$, where fluidlike aggregates grew for $t>0$ with inter-particle separations of order $a(\gg \xi)$. They observed that their average volume $V(t)$ and average length $L(t)$ were related by $V(t) \sim L(t)^{3} \propto t$ and the time-dependent scattered light intensity exhib- 
ited the Porod tail ${ }^{54}$. These behaviors are very different from those of fractal aggregates induced by the van der Waals interaction.

(2) Guo et $a l^{21}$ observed a fcc crystal formed by the aggregated particles with $a=52.5 \mathrm{~nm}$, where the lattice constant was $181 \mathrm{~nm}$ and the colloid volume fraction was 0.406 . In their case, the corner-to-face distance of the lattice was $2.46 a$ and the shortest face-to-face distance of the particles was $0.46 a=22 \mathrm{~nm}$. This separation distance is much longer than $\xi(=1-10 \mathrm{~nm})$. We thus propose a bridging scenario of crystal formation.

Acknowledgments. This work was supported by KAKENHI (No. 25610122) and Grants-in-Aid for Japan Society for Promotion of Science (JSPS) Fellows (Grants Nos. 241799 and 263111).

\section{Appendix: Renormalized free energy density}

To account for the statics in near-critical fluids, we use the renormalized local functional theory $\underline{47,48,55-57}$. As in the linear parametric model by Schofield et al $\stackrel{69}{ }$, we introduce a distance $w$ from the critical point in the $\tau$ - $\psi$ plane, in terms of which the overall critical behavior can be approximately described. We define $w$ by $\underline{\underline{47}}$

$$
w=\tau+C_{2} w^{1-2 \beta} \psi^{2},
$$

where $C_{2}$ is a constant. The coefficient $C$ in eqn (4) is set equal to $k_{B} T C_{1} w^{-\hat{\eta} \nu}$, where $C_{1}$ is a constant and $\hat{\eta}$ is the Fisher exponent.

The free energy density $f(\psi, \tau)$ is determined such that the combination $\xi^{3}[f(\psi, \tau)-f(0, \tau)] / k_{B} T$ is a universal function of $\tau /|\psi|^{1 / \beta}$ in accord with the two-scale factor universality 54.63 . Then, we obtain the correct exponent relations from the free energy $F=\int d \boldsymbol{r}\left[f+C|\psi|^{2} / 2\right]$, but the critical amplitude ratios ${ }^{54.63}$ are approximate depending on the choice of $C_{2}$. In this paper, we use the choice $C_{2}=\left(2 \pi^{2} / 3\right) C_{1} \xi_{0}$ in our previous work ${ }^{47}$. Then, we obtain $\xi_{0} / \xi_{0}^{\prime}=3.0$, while it is 1.9 from numerical analysis of the Ising criticality ${ }^{63}$.

In the original Schofield model 69 , the behavior of $f$ inside CX was not discussed. We assume $f$ inside CX (in the region $|\psi|<\psi_{\mathrm{cx}}$ and $\tau<0$ ) in the form,

$$
f(\psi, \tau)=f_{\mathrm{cx}}+k_{B} T_{c}\left(\psi^{2}-\psi_{\mathrm{cx}}^{2}\right)^{2} /\left(8 \chi_{\mathrm{cx}} \psi_{\mathrm{cx}}^{2}\right),
$$

where $f_{\mathrm{cx}}=f\left(\psi_{\mathrm{cx}}, \tau\right)$ and $\chi_{\mathrm{cx}}=k_{B} T_{c} /\left(\partial^{2} f / \partial \psi^{2}\right)$ on CX. Here, $\chi_{\mathrm{cx}} \sim|\tau|^{-\gamma}$ with $\gamma=(2-\hat{\eta}) \nu$. From eqn (4) and (A2) the surface tension is calculated as ${ }^{47}$

$$
\sigma=0.075 k_{B} T_{c} / \xi^{2}
$$

where $\xi=\xi_{0}^{\prime}|\tau|^{-\nu}$ is the correlation length on CX. See also Fig.3, where $\psi$ much exceeds $\psi_{\mathrm{cx}}$ near the colloid surfaces and tends to a value close to $-\psi_{\mathrm{cx}}$ in the bulk. Thus, use of eqn (A2) is essential in our theory.
${ }^{1}$ H. Tanaka, J. Phys.: Condens. Matter 13, 4637-4674 (2001).

2 P. D. Gallagher and J. V. Maher, Phys. Rev. A 46, 20122021 (1992).

3 H. Tanaka, A.J. Lovinger, and D.D. Davis, Phys. Rev. Lett. 72, 2581-2584 (1994).

4 A. Karim, J.F. Douglas, G. Nisato, D.W. Liu, and E.J. Amis, Macromolecules 32, 5917-5924 (1999).

5 V. V. Ginzburg, F. Qiu, M. Paniconi, G. Peng, D. Jasnow, and A. C. Balazs, Phys. Rev. Lett. 82, 4026-4029 (1999).

${ }^{6}$ M. Laradji and M. J. A. Hore, J. Chem. Phys. 12110641 (2004).

7 T. Araki and H. Tanaka, J. Phys.: Condens. Matter 20, (2008) 072101.

8 M.E. Fisher and P.G. de Gennes, C. R. Acad. Sci. Paris Ser. B 287207 (1978).

${ }^{9}$ M. Krech, Phys. Rev. E 56, 1642 (1997).

10 F. Schlesener, A. Hanke, and S. Dietrich, J. Stat. Phys. 110, 981-1013 (2003).

11 A. Gambassi, A. Maciolek, C. Hertlein, U. Nellen, L. Helden, C. Bechinger, and S. Dietrich, Phys. Rev. E 80, 061143 (2009).

12 A. Gambassi, J. Phys.: Conf. Ser. 161, 012037 (2009).

13 P. Hopkins, A.J. Archer, and R. Evans, J. Chem. Phys. 131, 124704 (2009).

14 D. Beysens and D. Estève, Phys. Rev. Lett. 54, 2123-2126 (1985).

15 V. Gurfein, D. Beysens, and F. Perrot, Phys. Rev. A 40:2543-2546 (1989).
16 M. L. Broide, Y. Garrabos, and D. Beysens, Phys. Rev. E 47, 3768 (1993).

17 D. Beysens, J. -M. Petit, T. Narayan, A. Kumar, and M. L. Broide, Ber. Bunsenges. Phys. Chem. 98, 382-388 (1994).

18 B.M. Law, J.- M. Petit, and D. Beysens, Phys. Rev. E, 57, 5782-5794(1998).

19 D. Beysens and T. Narayanan, J. Stat. Phys. 95, 997-1008 (1999).

20 P. D. Gallagher, M. L. Kurnaz, and J. V. Maher, Phys. Rev. A 46, 7750-7755 (1992).

21 H. Guo, T. Narayanan, M. Sztucki, P. Schall and G. Wegdam, Phys. Rev. Lett. 100, 188303 (2008).

22 D. Bonn, J. Otwinowski, S. Sacanna, H. Guo, G. Wegdam and P. Schall, Phys. Rev. Lett. 103, 156101 (2009).

23 T.J. Sluckin, Phys. Rev. A 41, 960-964 (1990).

24 H. Löwen, Phys. Rev. Lett. 74, 1028-1031 (1995).

${ }^{25}$ R. R. Netz Phys. Rev. Lett. 76, 3646-3649 (1996).

26 Y. Jayalakshmi and E. W. Kaler, Phys. Rev. Lett. 78, 1379-1382 (1997).

27 A.Gambassi and S.Dietrich, Phys. Rev. Lett. 105, 059601 (2010)

28 T. F. Mohry, A. Maciołek, and S. Dietrich, J. Chem. Phys. 136, 224903 (2012).

29 M. T. Dang, A. V. Verde, V. D. Nguyen, P. G. Bolhuis, and P. Schall, J. Chem. Phys. 139, 094903 (2013)

30 J. R. Edison, N. Tasios, S. Belli, R. Evans, R. van Roij, and M. Dijkstra, Phys. Rev. Lett. 114, 038301 (2015).

31 W. B. Russel, D. A. Saville, and W. R. Schowalter, Colloidal Dispersions (Cambridge University Press, Cam- 
bridge, UK, 1989).

32 C. Hertlein, L. Helden, A. Gambassi, S. Dietrich, and C. Bechinger, Nature 451, 172-175 (2008).

33 D. Bonn and D. Ross, Rep. Prog. Phys. 64, 1085-1163 (2001).

34 R. Evans, J. Phys.: Condens. Matter 2, 8989-9007 (1990).

35 L.D. Gelb, K.E. Gubbins, R. Radhakrishnan, and M. Sliwinska-Bartkowiak, Rep. Prog. Phys. 62, 1573-1658 (1999).

36 K. Binder, D. Landau, and M. Müller, J. Stat. Phys. 110, 1411-1514 (2003).

37 H. Nakanishi and M. E. Fisher, J. Chem. Phys. 78, 32793293 (1983).

38 R. Evans and U. M. B. Marconi, J. Chem. Phys. 86, 71387148 (1987).

39 H. T. Dobbs, G. A. Darbellay, and J. M. Yeomans, Europhys.Lett. 18, 439-444 (1992).

40 C. Bauer, T. Bieker, and S. Dietrich, Phys. Rev. E 62, 5324-5338 (2000).

41 C. D. Willett, M. J. Adams, S. A. Johnson, and J. P. K. Seville, Langmuir 16, 9396-9405 (2000).

42 H. Shinto, K. Uranishi, H. Miyahara, and K. Higashitani, J. Chem. Phys. 116, 9500-9509 (2002).

43 D. Andrienko, P. Patricio, and O. I. Vinogradova, J. Chem. Phys. 121, 4414-4423 (2004).

44 H.-J. Butt and M. Kappl, Adv. in Colloid and Interface Sci. 146, 48-60 (2009).

45 M.A. Hampton and A.V. Nguyen, Advances in Colloid and Interface Science 154, 30-55 (2010).

46 S. Herminghaus, Adv. Phys. 54, 221-261 (2005).

47 R. Okamoto and A. Onuki, J. Chem. Phys. 136, 114704 (2012).

48 R. Okamoto and A. Onuki, Phys. Rev. E. 88, 022309 (2013).

49 S. Yabunaka, R. Okamoto, and A. Onuki, Phys. Rev. E. 87, 032405 (2013).
50 A. Maciołek, A. Drzewiński,and R. Evans, Phys. Rev. E 64, 056137 (2001).

51 A. Furukawa, A. Gambassi, S. Dietrich, and H. Tanaka, Phys. Rev. Lett. 111 , 055701 (2013).

52 P.C. Hohenberg and B.I. Halperin, Rev. Mod. Phys. 49, 435-479 (1977).

53 K.Kawasaki and T. Ohta, Prog. Theor. Phys. 59, 362-374 (1978).

54 A. Onuki, Phase Transition Dynamics (Cambridge University Press, Cambridge, 2002).

55 M. E. Fisher and H. Au-Yang, Physica 101A, 255-264 (1980).

56 Z. Borjan and P. J. Upton, Phys. Rev. Lett. 81, 4911 (1998).

57 Z. Borjan and P. J. Upton, Phys. Rev. Lett. 101, 125702 (2008).

58 H. Tanaka and T. Araki, Phys. Rev. Lett. 85, 1338-1341 (2000).

59 Y. Fujitani, J. Phys. Soc. Jpn. 75, 013401 (2006).

60 J. Rudnick and D. Jasnow, Phys. Rev. Lett. 48, 1059-1062 (1982).

61 A. J. Liu and M. E. Fisher, Phys. Rev. A 40, 7202-7221 (1989).

62 B. M. Law, Prog. Surf. Sci. 66, 159-216 (2001).

63 A. J. Liu and M. E. Fisher, Physica A 156,35 (1989).

64 A. Pelissetto and E. Vicari, Phys. Rep. 368, 549 (2002).

65 M. Kardar and R. Golestanian, Rev. Mod. Phys. 71, 1233 (1999).

66 M. Krech and S. Dietrich, Phys. Rev. A 46, 1922 (1992),

67 M. Yada, J. Yamamoto, and H. Yokoyama, Phys. Rev. Lett. 92, 185501(2004);

${ }^{68}$ K. Takahashi, M. Ichikawa, and Y. Kimura, Phys. Rev. E 77, 020703R (2008).

69 P. Schofield, J.D. Lister, and J.T. Ho, Phys. Rev. Lett. 23, 1098-1101 (1969). 